EPJ Web of Conferences 59, 17012 (2013)

DOI: $10.1051 /$ epjconf/20135917012

(C) Owned by the authors, published by EDP Sciences, 2013

\title{
Efficient ion generation in laser-foil interaction
}

\author{
S. Kawata ${ }^{1, a}$, K. Takahashi ${ }^{1}$, D. Satoh ${ }^{1}$, D. Barada ${ }^{1}$, Y.Y. Ma ${ }^{2}$, Q. Kong ${ }^{3}$, \\ P.X. Wang ${ }^{3}$, W.M. Wang ${ }^{1,}{ }^{4}$, Y.T. Li ${ }^{4}$, Z.M. Sheng ${ }^{4,5}$, O. Klimo ${ }^{6}$, J. Limpouch ${ }^{6}$ \\ and A.A. Andreev ${ }^{7}$ \\ 1 Utsunomiya University, Utsunomiya 321-8585, Japan \\ 2 National University of Defense Technology, Changsha 410073, China \\ ${ }^{3}$ Fudan University, Shanghai 200433, China \\ 4 Inst. of Physics, CAS, Beijing 100190, China \\ 5 Shanghai Jiao Tong University, Shanghai 200240, China \\ ${ }^{6}$ Czech Technical University, CZ-182 121 Praha 8, Czech Republic \\ 7 Inst. of Laser Physics, St. Petersburg 199034, Russia
}

\begin{abstract}
A remarkable improvement is presented on the energy conversion efficiency from laser to protons in a laser-foil interaction by particle simulations. The total laser-proton energy conversion efficiency from laser to protons becomes $16.7 \%$, though a conventional plane foil target serves a rather low efficiency. In our 2.5-dimensional particle-in-cell simulations the Al multihole structure is also employed, and the laser absorption ratio reaches $71.2 \%$. The main physical reason for the enhancement of the conversion efficiency is a reduction of the laser reflection at the target surface area;
\end{abstract}

\section{INTRODUCTION}

Ion beams are useful for basic particle physics, medical therapy, controlled nuclear fusion, high-energy sources, and so on [1-8]. The energy of ions, which are accelerated in an interaction between an intense laser pulse and a thin foil target, has been over $\mathrm{MeV}$ [9-24]. The ion acceleration in the laser-foil interaction is expected to be a new method of ion acceleration. The issues in the laser ion acceleration include ion beam collimation [9, 13], ion energy spectrum control, ion production efficiency [21-23], etc. In this paper we focus on the energy conversion efficiency from laser to protons in the laser-ion acceleration, and so far the energy conversion efficiency was still relatively low in actual experiments.

When an intense short-pulse laser illuminates a thin foil target, electrons are first accelerated by the laser, and oscillate or move around the thin foil. The electrons form a high current and generate a magnetic field. In the laser-foil interaction, the ion dynamics is affected directly by the behavior of the electrons $[12,13]$. The electrons form a strong electric field, and the ions are accelerated by the electric field. When a foil target has backside multiholes at the target rear side (see Fig. 1(c)) and the hole size is the order of the laser spot size, the holes help to produce a collimated ion beam [13].

On the other hand, the foil target surface reflects a significant part of the laser energy. The energy conversion efficiency from laser to protons tends to be low. The subwavelength-multiholes transpiercing the planar target enhance the laser-proton energy conversion efficiency [21-23]. The subwavelength microstructured targets [21-25] are propitious to enhance the laser energy absorption. In this paper

\footnotetext{
ae-mail: kwt@cc.utsunomiya-u.ac.jp
}

This is an Open Access article distributed under the terms of the Creative Commons Attribution License 2.0, which permits unrestricted use, distribution, and reproduction in any medium, provided the original work is properly cited. 


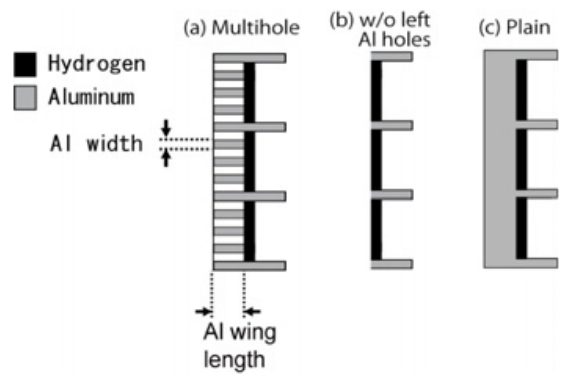

Figure 1. Thin-foil targets: (a) a multihole target (b) a thin foil target without an Al hole layer and (c) a planar target (a conventional target with a plain Al layer).

we employ the subwavelength-multihole microstructure to increase the laser-proton energy transfer efficiency at the target rear side.

Throughout the paper wider holes at the rear side are employed to ensure the collimated proton beam generation [13] (see Figs. 1(a)-(c)). The Al width in the right hand side is $1.0 \lambda$ for the proton beam collimation.

\section{EFFICIENT LASER ENERGY CONVERSION TO IONS IN LASER HOLE-TARGET INTERACTION}

We perform 2.5-dimensional ( $\mathrm{x}, \mathrm{y}, \mathrm{v}_{x}, \mathrm{v}_{y}, \mathrm{v}_{z}$ ) particle-in-cell simulations. Figure 1(a) shows a conceptual diagram of the multihole target. The laser intensity is $I=1.0 \times 10^{20} \mathrm{~W} / \mathrm{cm}^{2}$, the laser spot diameter is $4.0 \lambda$, and the pulse duration is $20 \mathrm{fs}$. The laser transverse profile is in the Gaussian distribution, and the laser temporal profile is Gaussian. The laser wave length is $\lambda=1.053 \mu \mathrm{m}$. The ionization degree of $\mathrm{Al}$ layer substrate is 11 , and the Al layer density is the solid density.

We study the role of the Al width and the Al wing length in the multihole target shown in Fig. 1(a). At the target rear side the additional wider holes are installed to ensure the proton beam collimation based on our previous study [13] throughout the paper. Thin foil tailored targets, which have a subwavelength structure, enhance the laser energy absorption [21-24].

Figure 1(a) shows the tailored multihole target. Firstly the Al width of the multiholes at the laser side is changed from $0.1 \lambda$ to $0.5 \lambda$. For a comparison, we also simulate the target which has no multihole $\mathrm{Al}$ (see Fig. 1(b)). At the subwavelength-structured Al surface, seen by the illuminating laser, the laser is partly reflected. When a laser interacts with a wire target, a large number of electrons are expelled out from the target and accelerated efficiently. Therefore, when the Al is thinner in Fig. 1(a), the laser enters into the target $\mathrm{Al}$ microstructure and generates hot electrons effectively. So the thinner Al-layer target produces the higher acceleration electric field. Figure 2 shows the total energy histories of the proton for each case. When the Al width is $0.1 \lambda$, the total energy of the protons reaches about $4.5 \times 10^{3} \mathrm{~J} / \mathrm{m}$ in the multihole target at $300 \mathrm{fs}$. The maximum proton kinetic energy is $12.2 \mathrm{MeV}$. For the other cases it is $9.51 \mathrm{MeV}$ for $0.3 \lambda$ and $7.48 \mathrm{MeV}$ for $0.5 \lambda$. When the $\mathrm{Al}$ width becomes thin, the $\mathrm{Al}$ surface area seen by the laser is reduced and the laser reflection is also reduced. However, if the target has no $\mathrm{Al}$ microstructure at the laser side, it just becomes a plain target. The energy laser-proton conversion efficiency is $13.3 \%$ for $0.1 \lambda, 8.67 \%$ for $0.3 \lambda$ and $6.28 \%$ for $0.5 \lambda$, though it is $5.74 \%$ for the target in Fig. 1(b). Moreover, the laser Al-ion energy conversion efficiency is $38.0 \%$ for $0.1 \lambda$. The optimal width of the $\mathrm{Al}$ microstructure in Fig. 1(a) must be the order of the skin depth to reduce the laser reflection as low as possible. The simulation results present that the proton total number for the target of $0.1 \lambda$ is about 2.0 times large compared with that for $0.5 \lambda$. In the conventional target shown in Fig. 1(c) the laser-proton energy conversion efficiency was $1.81 \%$. 


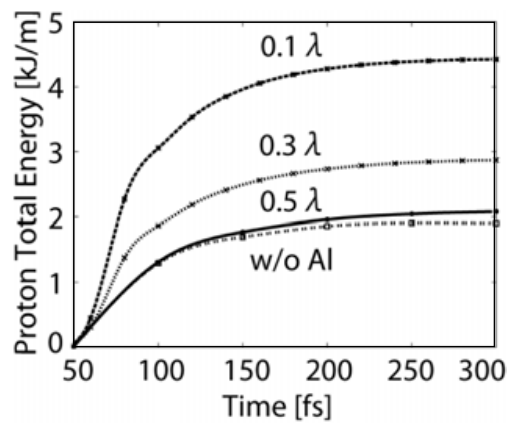

Figure 2. Total energy histories of the protons accelerated for the structured target with the Al width in $0.5 \lambda, 0.3 \lambda$ and $0.1 \lambda$ and for the target without the mictostructure in Fig. 1(b).
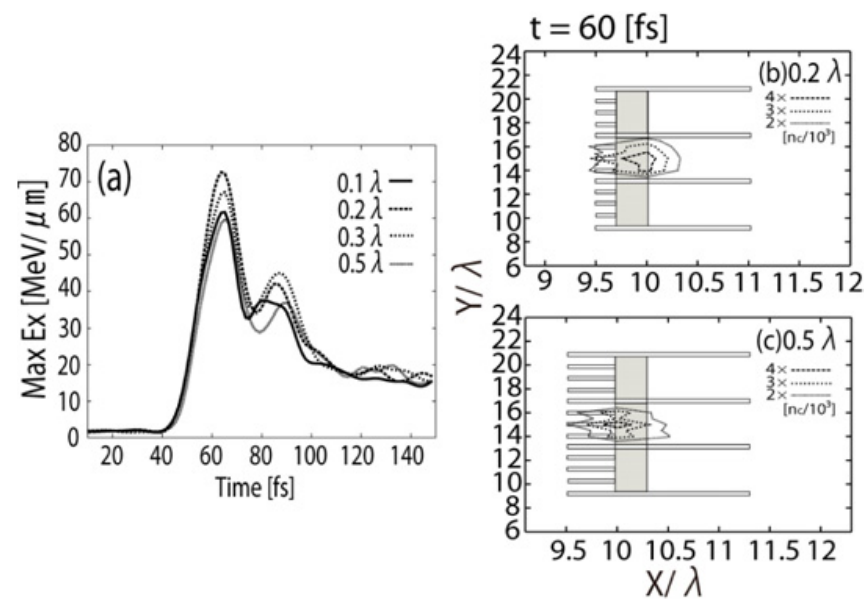

Figure 3. (a) The maximal acceleration electric field $E x$ for the multihole target, and distributions of the high-energy electron density at (b) $0.2 \lambda$ and (c) $0.5 \lambda$.

The results in this paper demonstrate a significant effect of the $\mathrm{Al}$ microstructure width on the ion generation efficiency. As discussed above, the physics of the increase in the proton generation efficiency comes from mainly the reduction of the laser reflection at the left surface area [21,22]. The reduction of the $\mathrm{Al}$ reflection area, that is the reduction of the $\mathrm{Al}$ width in Fig. 1(a), results in the significant increase in the laser energy conversion to the protons. In addition, the microstructure holes contribute to an increase of the laser interaction surface area, and laser also reflects multiple times inside the holes.

We also examine the role of the $\mathrm{Al}$ wing length on the laser-ion energy conversion. We change the Al wing length of the multihole target from $0.1 \lambda$ to $0.5 \lambda$ (see Fig. 1(a)). At $0.2 \lambda$ protons obtain the larger energy from the laser. At the $\mathrm{Al}$ wing length of $0.2 \lambda$, the total energy of the protons reaches the maximum and about $5.5 \times 10^{3} \mathrm{~J} / \mathrm{m}$ in the multihole target. The energy conversion efficiency from the laser to the protons becomes $16.7 \%$. The maximum proton kinetic energy is $12.4 \mathrm{MeV}$ for the case of $0.2 \lambda, 10.4 \mathrm{MeV}$ for $0.1 \lambda, 13.0 \mathrm{MeV}$ for $0.3 \lambda$ and $12.2 \mathrm{MeV}$ for $0.5 \lambda$ at $300 \mathrm{fs}$. The energy conversion efficiency is $38.7 \%$ for laser Al-ions and $39.0 \%$ for laser-electrons. The laser absorption ratio is $71.2 \%$ for $0.2 \lambda$. Figure 3 shows (a) the maximal acceleration electric field $E \mathrm{x}$ for the multihole target, and the distributions of the high-energy electron density at (b) $0.2 \lambda$ and (c) $0.5 \lambda$. The hot-electron highdensity cloud is well located at the right space of the proton layer in the case of $0.2 \lambda$ for the effective proton acceleration. 


\section{EPJ Web of Conferences}

When the $\mathrm{Al}$ wing length is too short, the total electron number in the $\mathrm{Al}$ layer is reduced to create the proton acceleration field at the target rear side. The proton acceleration field, created by the hot electrons at the rear side, is produced by the hot electrons. When the total number of the electrons, which are mainly originated from the microstructured $\mathrm{Al}$ at the laser side, becomes small, the acceleration field at the rear side becomes weak. On the other hand, when the Al wing length is too long, the hot electron cloud cannot fully reach the target rear side (see Fig. 3(c)). In this case with the long Al wing the Al layer originally contains a sufficient number of electrons, and the sufficient number of hot electrons appears. However, the hot electron cloud experiences the interaction with the target plasma for a longer distance than that in the optimal case, and does not reach the correct position of the target rear side to create a strong acceleration electric field at the target rear side, as presented in Fig. 3(c). However, for the optimal case, that is, the case with the Al optimal wing length of $0.2 \lambda$, the hot-electron cloud location is appropriate to create the high acceleration field $E \mathrm{x}$ for the effective proton acceleration as shown in Fig. 3(b).

\section{CONCLUSIONS}

In this paper, we presented an efficient energy conversion method from laser to ions in laser foil interaction. We investigated and clarified the role of the hole width and width of the tailored multihole target at the laser side in the laser-proton energy conversion. The conversion efficiency was enhanced significantly to $16.7 \%$ in the optimal microstructured target from a few percent in a planar target without the microstructure. For practical applications of the laser-ion accelerator, the issues contain the efficient ion generation as studied in this paper, the ion beam quality improvement in the energy spectrum control including a mono-energy ion beam generation, neutralized or unneutralized ion beam transportation for a long distance, laser multi-stage post acceleration, etc. The multihole target presented in this paper may serve a new way to create proton beams efficiently in the future laser proton accelerator.

This work is partly supported by MEXT, JSPS, ILE/Osaka Univ. and CORE (Center for Optical Research and Education, Utsunomiya university, Japan)

\section{References}

[1] S. V. Bulanov and V. S. Khoroshkov, Plasma Phys. Rep. 28, 453 (2002)

[2] M. Roth, T. E. Cowan, M. H. Key, S. P. Hatchett, C. Brown, W. Fountain, J. Johnson, D. M. Pennington, R. A. Snavely, S. C. Wilks, K. Yasuike, H. Ruhl, F. Pegoraro, S. V. Bulanov, E. M. Campbell, M. D. Perry and H. Powell, Phys. Rev. Lett. 86, 436 (2001)

[3] M. Kaluza, J. Schreiber, M. I. K. Santala, G. D. Tsakiris, K. Eidmann, J. Meyer-ter-Vehn and K. J. Witte, Phys Rev. Lett. 93, 045003 (2004)

[4] Y. Sentoku, T. V. Liseikina, T. Zh. Esirkepov, F. Califano, N. M. Naumova, Y. Ueshima, V. A. Vshivkov, Y. Kato, K. Mima, K. Nishihara, F. Pegoraro and S. V. Bulanov, Phys. Rev. E 62, 7271 (2000)

[5] S. Ter-Avetisyan, M. Schnürer, P. V. Nickles, M. Kalashnikov, E. Risse, T. Sokollik, W. Sandner, A. Andreev and V. Tikhonchuk, Phys. Rev. Lett. 96, 145006 (2006)

[6] A. B. Langdon and B. F. Lasinski, Methods Comput. Phys. 16, 327 (1976)

[7] A. A. Andreev, K. Yu. Platonov, T. Okada and S. Toraya, Phys. Plasmas 10, 220 (2003)

[8] S. V. Bulanov, T. Zh. Esirkepov, J. Koga, T. Tajima and D. Farina, Plasma Phys. Rep. 30, 18 (2004) 
[9] M. Nakamura, S. Kawata, R. Sonobe, S. Miyazaki, Q. Kong and T. Kikuchi, J. Appl. Phys. 101, 113305 (2007)

[10] S. V. Bulanov, T. Zh. Esirkepov, F. Califano, Y. Kato, T. V. Lisekina, K. Mima, N. M. Naumova, K. Nishihara, F. Pegorano, H. Ruhl, Y. Sentoku and Y. Ueshima, JETP Lett. 71, 407 (2000)

[11] T. P. Yu, Y. Y. Ma, M. Chen, F. Q. Shao, M. Y. Yu, Y. Q. Gu and Y. Yin, Phys. Plasmas 16, 033112 (2009)

[12] T. Nakamura and S. Kawata, Phys. Rev. E 67, 026403 (2003)

[13] R. Sonobe, S. Kawata, S. Miyazaki, M. Nakamura and T. Kikuchi, Phys. Plasmas 12, 073104 (2005)

[14] T. Nakamura, S. V. Bulanov, T. Z. Esirkepov, and M. Kando: Phys. Rev. Lett. 105, 135002 (2010)

[15] S. P. Hatchett et al., Phys. Plasmas 7, 2076 (2000)

[16] M. Borghesi, A. J. Mackinnon, D. H. Campbell, D. G. Hicks, S. Kar, P. K. Patel, D. Price, L. Romagnani, A. Schiavi and O. Willi, Phys. Rev. Lett. 92, 055003 (2004)

[17] F. Lindau, O. Lundh, A. Persson, P. McKenna, K. Osvay, D. Batani and C.-G. Wahlström, Phys. Rev. Lett. 95, 175002 (2005)

[18] T. Zh. Esirkepov, S. V. Bulanov, K. Nishihara, T. Tajima, F. Pegoraro, V. S. Khoroshkov, K. Mima, H. Daido, Y. Kato, Y. Kitagawa, K. Nagai and S. Sakabe, Phys. Rev. Lett, 89, 175003 (2002)

[19] T. Esirkepov, M. Borghesi, S. V. Bulanov, G. Mourou and T. Tajima, Phy. Rev. Lett. 92, 175003 (2004)

[20] A. P. L Robinson, A. R. Bell and R. J. Kingham, Phys. Rev. Lett. 96, 035005 (2006)

[21] W. M. Wang, Z. M. Sheng and J. Zhang, Phys. Plasmas 15, 030702 (2008)

[22] Y. Nodera, S. Kawata, N. Onuma, J. Limpouch, O. Klimo and T. Kikuchi, Phys. Rev. E 78, 046401 (2008)

[23] Y. Y. Ma, Z. M. Sheng, J. Zhang, Y. T. Li, W. W. Chang, X. H. Yuan, M. Chen and J. Zheng, Phys. Plasmas 13, 110702 (2006)

[24] S. Kahaly, S. K. Yadav, W. M. Wang, S. Sengupta, Z. M. Sheng, A. Das, P. K. Kaw and G. R. Kumar, Phys. Rev. Lett. 101, 145001 (2008)

[25] T. Palchan, S. Pecker, Z. Henis, S. Eisenmann, and A. Zigler, Appl. Phys. Lett. 90, 041501 (2007) 